\title{
Indices of Obesity and Cardiovascular Risk Factors in British Women
}

\author{
See Kwok ${ }^{a}$ Patrick McElduff ${ }^{b}$ David W. Ashton ${ }^{c}$ Gordon D.O. Lowe ${ }^{d} \quad$ D. Wood \\ Stephen E. Humphries ${ }^{e}$ Valentine Charlton-Menys ${ }^{a}$ Paul N. Durrington ${ }^{\mathrm{a}}$ \\ a School of Clinical and Laboratory Sciences, University of Manchester, UK \\ ${ }^{\text {b}}$ Datapharm Australia, Drummoyne, NSW, Australia \\ ${ }^{c}$ National Heart \& Lung Institute Faculty of Medicine, Imperial College of Science Technology \& Medicine, \\ Charing Cross Hospital Cardiology Department, London, \\ dUniversity of Glasgow, Department of Medicine, Glasgow Royal Infirmary, Glasgow, \\ e Centre for Cardiovascular Genetics, Royal Free \& University College Medical School, Division of Medicine, The Rayne Building, London, UK
}

\section{Key Words}

Blood pressure $\cdot$ C-reactive protein - Fibrinogen . High density lipoproteins - Low density lipoproteins . Obesity · Triglycerides

\section{Summary}

Background: Obesity increases cardiovascular risk through effects on blood pressure, lipoproteins, coagulation factors and inflammatory cytokines, but in women variation in fat distribution complicates these relationships. Central (maletype or visceral) obesity confers greater risk than the more generalised (female) type. This is recognised by the metabolic syndrome which employs waist circumference rather than body mass index (BMI). We examined the relationships of several indices of fat distribution with cardiovascular risk factors in a large cohort of UK women. Material and Methods: 13,389 female department store employees aged 30-65 years not receiving exogenous hormones completed a health questionnaire. Their blood pressure, weight, height, waist and hip circumference, serum cholesterol low density lipoprotein cholesterol (LDL-C), high density lipoprotein cholesterol (HDL-C), triglycerides, C-reactive protein (CRP) and plasma fibrinogen were measured. Results: There was a progressive rise in blood pressure, total cholesterol, LDL-C, triglycerides, fibrinogen and CRP with age. After adjustment for these age effects, BMI was most closely related to blood pressure, whereas the waist to height ratio (WHTR) correlated more closely with the other risk factors than BMI, waist circumference or waist to hip ratio (WHPR). Conclusions: Inclusion of height in the definition of metabolic syndrome will produce a clearer association between waist circumference and cardiovascular risk factors. Hypertension may be linked to the metabolic syndrome by its association with general obesity rather than specifically by central obesity.

\section{Introduction}

Epidemiological studies have demonstrated a gender difference in the incidence of atherosclerotic cardiovascular disease (CVD), with women, particularly younger women, at much lower risk of developing CVD than age-matched men [1]. Because of this, women have been under-represented in many of the major studies on cardiovascular risk factors [2]. However, CVD is the largest single cause of death in women worldwide [3], and, when prevalent morbidity rates are considered, the longer survival of women into old age means that the absolute number of affected women exceeds that of men [4]. Risk factors for CVD are therefore relevant for women, and studies of large female cohorts continue to be important.

The distribution of body fat differs in obese men and women. Men almost invariably tend to accumulate fat intra-abdominally (central, android or visceral obesity). Women who become obese may follow this pattern, but commonly, instead, develop adiposity in the gluteo-femoral region (gynecoid obesity). This has been thought in part to result in the lower cardiovascular risk in women, because the intra-abdominal or visceral collection of fat in men is more metabolically active and causes more marked 'insulin' resistance and dyslipidaemia [5, 6]. When women do develop central obesity, as is typically the case if they have type 2 diabetes or metabolic syndrome (MS), some of their protection against CVD is lost. The recognition of central obesity may thus be particularly important in identifying women at high CVD risk. Central adiposity causes clustering of cardiovascular risk factors [7] and is the major determinant of MS, a constellation of risk indicators including decreased high density lipoprotein cholesterol (HDL-C), raised triglycerides, insulin resistance and high blood pressure, predisposing to increased cardiovascular hazard $[8,9]$. Raised

\begin{tabular}{ll}
\hline KARGER & ๑ 2008 S. Karger GmbH, Freiburg \\
Fax +497614520714 & Accessible online at: \\
$\begin{array}{l}\text { E-mail Information@Karger.de } \\
\text { www.karger.com }\end{array}$ & www.karger.com/ofa
\end{tabular}

Prof. Dr. Paul N. Durrington

Cardiovascular Research Group, School of Clinical and Laboratory Sciences

Core Technology Facility (3rd Floor), University of Manchester

46 Grafton Street, Manchester M13 9NT, UK

Tel. +44 161 275-1201, Fax -1183

pdurrington@manchester.ac.uk 
circulating C-reactive protein (CRP) and fibrinogen are other cardiovascular risk factors which could potentially be included in MS $[8,9]$. The increase in the prevalence of MS closely correlates with the rise in obesity worldwide and affects both men and women [3].

There are various indices for the estimation of obesity including body mass index (weight:height ${ }^{2}$ ) (BMI), waist circumference (WC), waist to hip ratio (WHPR) and waist to height ratio (WHTR). The most widely used clinical index of obesity is the BMI, but this does not discriminate central from more generalised obesity. Guidelines recently proposed for CVD prevention include MS in CVD risk estimation and make WC the preferred indicator of central obesity [10-12]. It is particularly important to test this proposal critically in women, because of their greater heterogeneity in fat distribution. Using data from the United Kingdom Women's Heart Study (UKWHS), a large prospective study of cardiovascular risk factors in a population of women, we examine the relationships of the various obesity indices (BMI, WC, WHPR, WHTR) and age with blood pressure, total cholesterol, HDLC, triglycerides, low density lipoprotein cholesterol (LDL-C), CRP and fibrinogen.

\section{Material and Methods}

\section{Subjects}

Female employees aged 30-65 years from 107 Marks and Spencer stores in the UK were invited to participate in the UKWHS. Women were eligible for the study if they had been employed for a minimum of 6 months and were not on maternity or sick leave at the time of recruitment. Ethical approval for the study was granted by a central ethics committee. Each participant also gave her individual informed consent to the study. About 8-10 weeks prior to screening, each of the stores was visited by one of a team of eight specially trained nurses who gave 30-min presentations on the UKWHS. Women who consented to the study were asked to complete a questionnaire and attend for a health check at their place of work between June 1988 and July 1991.

The questionnaire included information on personal medical history, current medications including hormone use and smoking habits. The health check was carried out by specially trained nurses. Height and weight were measured using a Seca model 707 digital scales with stadiometer (Vogel and Halke, Hamburg, Germany), and BMI calculated. WC was measured midway between the top of the iliac crest and the lower rib margin. Hip circumference was the measurement around the widest part of the hips. After resting in the sitting position for $5 \mathrm{~min}$, blood pressure was measured using a standard mercury sphygmomanometer. Korotkoff phase V was used as diastolic blood pressure. Blood pressure readings were recorded at the beginning and at the end of the screening session, and the mean of the 2 readings calculated. Screening sessions were conducted throughout the working day, and thus the majority were not fasting. Venous blood samples were taken for measurements of total cholesterol, HDL-C, triglycerides, CRP and fibrinogen.

In all 21,310 women were screened from a female workforce of approximately 27,000. Exogenous hormone use is known to modify cardiovascular risk factors, thus we excluded women who were taking the contraceptive pill or hormone replacement therapy $(n=7,776)$. We also excluded women who reported personal history of CVD $(n=67)$ and diabetes $(n=78)$. Data from the remaining women were analysed. There were 13,389 height, weight and blood pressure measurements recorded,
11,184 results for CRP and 11,042 with complete results for serum cholesterol LDL-C, HDL-C and triglycerides. Fibrinogen was measured in a subset of women $(n=5,929)$.

\section{Laboratory Methods}

Assays of lipoproteins and CRP were carried out in the Lipid Research Laboratory at Manchester University. Serum and EDTA plasma was obtained by centrifugation at $2,056 \times g(\operatorname{gmax})$ for $15 \mathrm{~min}$ at $4{ }^{\circ} \mathrm{C}$. Aliquots $(0.5 \mathrm{ml})$ were frozen in polypropylene tubes before storage at $-80{ }^{\circ} \mathrm{C}$ for an average of 10 years (range 9-11 years). Serum cholesterol was determined using the CHOD-PAP method (reagents from Horiba ABX Diagnostics, Shefford, UK) with an intra-assay coefficient of variation (CV) of $0.9 \%(n=8)$ and an inter-assay CV of $4.2 \%(n=6)$, and HDL-C was determined using a direct 2nd generation homogenous PEGME method (Roche Diagnostics, Lewes, UK) with intra- and inter-assay CVs of $1.5 \%$ in each case. A Cobas Mira analyser (Hoffman la Roche Ltd, Basel, Switzerland) was used for cholesterol and HDL-C assays. LDL-C was calculated using the Friedewald equation [13]. CRP was measured using a high-sensitivity assay with reagents and a BNII analyzer from DadeBehring, Milton Keynes, UK. The intra- and inter-assay CVs for the CRP assay were 3.9 and $4.6 \%$, respectively. Assays of fibrinogen were carried out at Glasgow Royal Infirmary using a Dade-Behring nephelometer and reagents. The intra-and inter-assay CVs for the fibrinogen assay were 2.6 and $5.3 \%$, respectively.

\section{Statistical Analysis}

Statistical analyses were made using Stata statistical package version 8 (StataCorp LP, College Station, TX, USA). Means and 95\% confidence intervals (CI) were calculated for all anthropometric and biochemical measures as well as for the log transformed values of CRP and triglycerides. The estimated increase in each of the anthropometric and biochemical measures associated with increasing age was estimated using linear regression with the measure of interest as the outcome variable and age as the predictor. The results of the regression models are presented as the average increase for each 10-year increase in age, which was calculated by multiplying the coefficients for age from the regression models by 10 . Additional linear regression models were fitted to examine which of the obesity indices was the 'best' predictor of each of the biochemical measurements.

Risk factors are reported as mean \pm standard deviation (SD), but also as median and the 90th percentile (or in the case of HDL the 10th percentile) to be consistent with earlier reports $[14,15]$ in which people at high cardiovascular risk from a single risk were defined in terms of those in the most adverse $10 \%$ of the population, although currently therapeutic decisions tend to be based on estimates of overall cardiovascular risk [10-12]. Because the indices of obesity to be compared employed different units and because their standard deviations differ, comparison of their impact on risk factors would be meaningless based on measurement units or on I-SD change. They were therefore converted to standard scores (Z-scores) according to the formula

$$
Z=\frac{X-\text { mean }}{\text { standard deviation }}
$$

All then had a mean value of zero and SD of unity.

\section{Results}

The age of the women in the study ranged from 30 to 65 years. $10 \%$ of women were aged $30-34(\mathrm{n}=1,292), 13 \%$ were aged 35-39 years $(\mathrm{n}=1,773)$ and $18 \%$ were aged $40-44$ years $(\mathrm{n}=2,477)$. More than a quarter $(26 \%)$ of the participants 

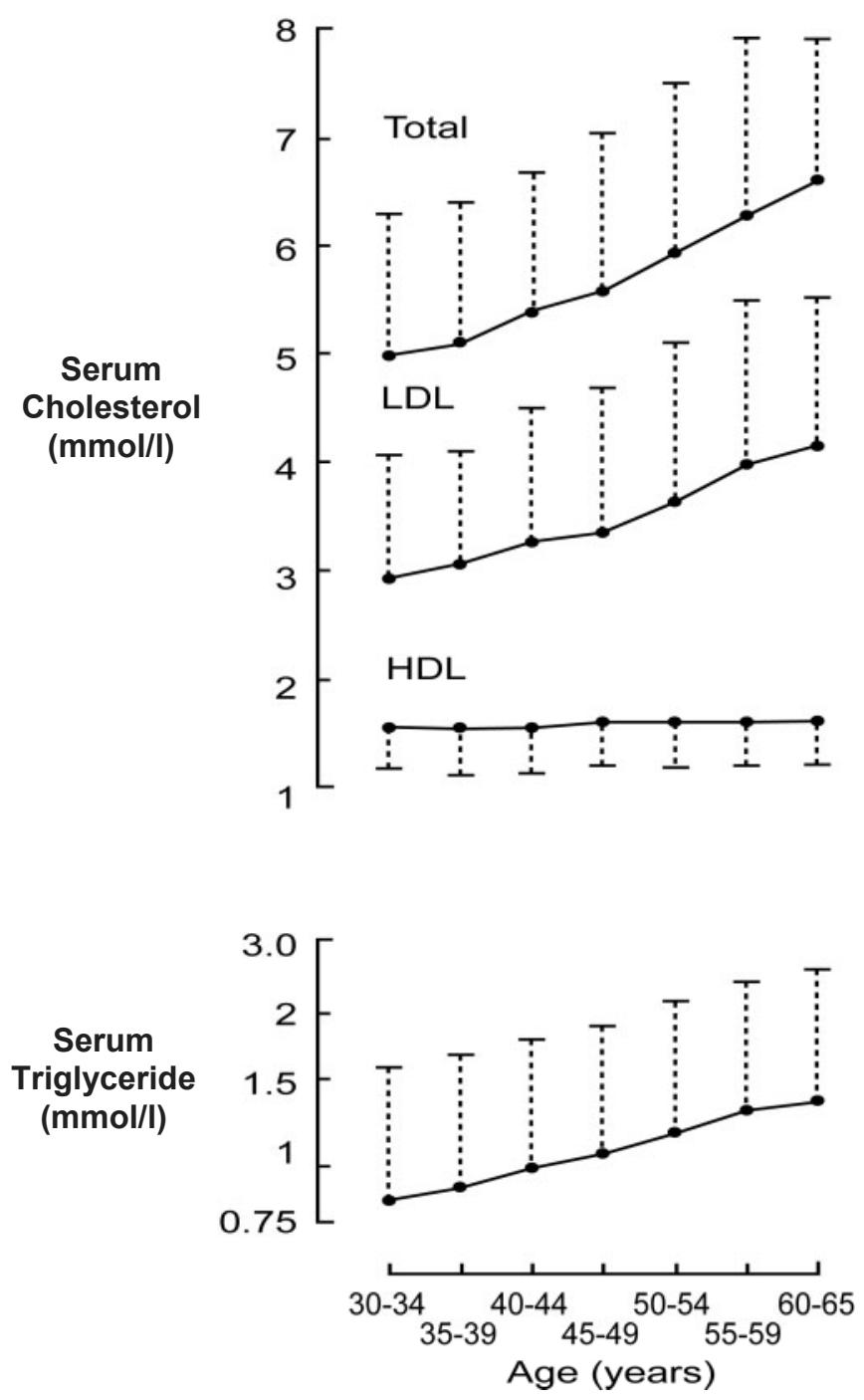

Fig. 1. The serum cholesterol, LDL-C, HDL-C and serum triglyceride concentrations in British women as a function of age. Values are means (geometric mean in the case of triglyceride). The upward vertical lines indicate the 90th percentile (the downward vertical lines indicate the 10th percentile for HDL cholesterol).

were aged $45-49$ years $(n=3,452), 18 \%$ were aged $50-54$ years $(\mathrm{n}=2,384), 12 \%$ were aged $55-59$ years $(\mathrm{n}=1,599)$ and $3 \%$ were over 60 years $(n=412)$. Age was found to have a significant effect on all the measured parameters except HDL-C (table 1). All indices of obesity increased with advancing age. Figure 1 shows the distribution of lipoproteins for the different age groups. The values for total cholesterol, LDL-C and triglycerides increased with age. Figure 2 shows the increase in fibrinogen, CRP and blood pressure with age. The 4 anthropometric measures had a similar level of association with each of the lipid measures used in this study, but after adjusting for age, WHTR in this cohort explained more of the variation in all lipid measures, fibrinogen and CRP than BMI, WC or WHPR (table 2). BMI, however, explained more of the variation in systolic and diastolic blood pressure than any of the
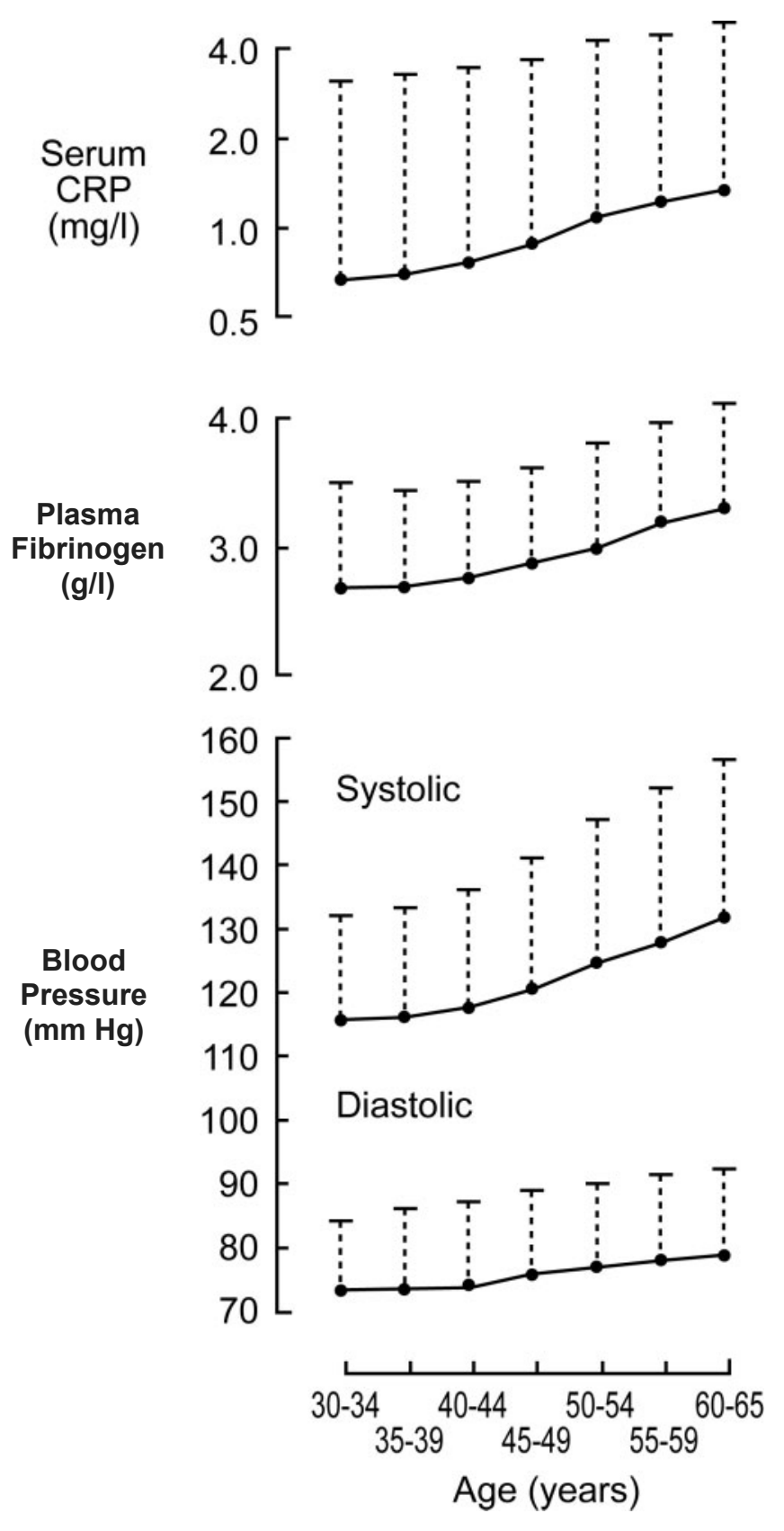

Fig. 2. The serum CRP and plasma fibrinogen levels and the systolic and diastolic blood pressure as a function of age in British women. Values are means and the vertical lines are 90th percentile.

other 3 anthropometric measures, and WHPR significantly less of the variation in CRP and fibrinogen than the other measures.

\section{Discussion}

We report that WHTR is more closely associated with cardiovascular risk factors in women with the exception of blood pressure which correlates best with BMI. For none of the risk factors studied, was WC the most closely related obesity 
Table 1. Mean level (standard deviation) of each anthropometric and biochemical measure

\begin{tabular}{|c|c|c|c|c|c|c|c|}
\hline & \multicolumn{7}{|l|}{ Age, years } \\
\hline & $30-34$ & $35-39$ & $40-44$ & $45-49$ & $50-54$ & $55-59$ & $60-64$ \\
\hline Women, $\mathrm{n}$ & 1,292 & 1,773 & 2,477 & 3,452 & 2,384 & 1,599 & 412 \\
\hline $\mathrm{BMI}, \mathrm{kg} / \mathrm{m}^{2}$ & $24(4)$ & $25(4)$ & $25(4)$ & $25(4)$ & $26(4)$ & $26(4)$ & $26(4)$ \\
\hline $\mathrm{WC}, \mathrm{cm}$ & $73(9)$ & $74(9)$ & $75(9)$ & $76(9)$ & $77(9)$ & $79(9)$ & $79(9)$ \\
\hline WHPR & $0.74(0.05)$ & $0.74(0.05)$ & $0.74(0.05)$ & $0.75(0.06)$ & $0.76(0.06)$ & $0.77(0.06)$ & $0.78(0.06)$ \\
\hline WHTR & $0.45(0.06)$ & $0.46(0.06)$ & $0.47(0.06)$ & $0.47(0.06)$ & $0.48(0.06)$ & $0.49(0.06)$ & $0.50(0.06)$ \\
\hline Systolic BP, mm Hg & $116(11)$ & $117(12)$ & $119(13)$ & $123(15)$ & $126(16)$ & $130(17)$ & $133(18)$ \\
\hline Diastolic BP, mm Hg & $74(9)$ & $74(9)$ & $75(9)$ & $76(10)$ & $77(10)$ & $78(10)$ & $79(10)$ \\
\hline Total cholesterol, $\mathrm{mmol} / \mathrm{l}$ & $5.1(1.0)$ & $5.2(1.0)$ & $5.5(1.0)$ & $5.7(1.1)$ & $6.1(1.2)$ & $6.4(1.2)$ & $6.5(1.1)$ \\
\hline LDL-C, mmol/1 & $3.0(0.9)$ & $3.1(0.9)$ & $3.3(0.9)$ & $3.5(0.9)$ & $3.8(1.3)$ & $4.1(1.1)$ & $4.2(1.0)$ \\
\hline HDL-C, mmol/l & $1.59(0.36)$ & $1.58(0.38)$ & $1.59(0.38)$ & $1.64(0.39)$ & $1.65(0.40)$ & $1.63(0.38)$ & $1.61(0.37)$ \\
\hline Total cholesterol/HDL-C & $3.3(0.9)$ & $3.5(1.2)$ & $3.6(1.1)$ & $3.7(2.3)$ & $3.9(1.4)$ & $4.11(1.2)$ & $4.2(1.2)$ \\
\hline Triglycerides, mmol/1 & $0.99(0.52)$ & $1.05(0.53)$ & $1.13(0.54)$ & $1.21(0.63)$ & $1.39(1.83)$ & $1.45(0.72)$ & $1.54(0.81)$ \\
\hline $\mathrm{CRP}, \mathrm{mg} / \mathrm{l}$ & $1.41(2.71)$ & $1.59(3.58)$ & $1.60(2.92)$ & $1.75(3.10)$ & $2.00(3.14)$ & $2.20(3.88)$ & $2.29(2.98)$ \\
\hline Fibrinogen, g/l & $2.77(0.55)$ & $2.73(0.56)$ & $2.82(0.55)$ & $2.90(0.55)$ & $3.04(0.57)$ & $3.22(0.57)$ & $3.32(0.69)$ \\
\hline
\end{tabular}

$\mathrm{BP}=$ Blood pressure

Table 2. The change in the mean $(95 \% \mathrm{CI})$ level of the cardiovascular risk factors associated with a 1-unit increase in the Z-score for each index of obesity, after adjusting for age in linear regression models

\begin{tabular}{|c|c|c|c|c|}
\hline \multirow[t]{2}{*}{ Risk factors } & \multicolumn{4}{|c|}{ Change in risk factor for 1-unit increase in the Z-score for each index of obesity } \\
\hline & $\mathrm{BMI}, \mathrm{kg} / \mathrm{m}^{2}$ & $\mathrm{WC}, \mathrm{cm}$ & WHPR & WHTR \\
\hline Total cholesterol, mmol/1 & $0.111(0.091,0.132)$ & $0.136(0.115,0.156)$ & $0.142(0.121,0.162)$ & $0.156(0.135,0.176)$ \\
\hline LDL-C mmol/l & $0.129(0.109,0.148)$ & $0.146(0.127,0.165)$ & $0.144(0.124,0.163)$ & $0.164(0.144,0.183)$ \\
\hline $\mathrm{HDL}-\mathrm{C}, \mathrm{mmol} / \mathrm{l}$ & $-0.095(-0.102,-0.088)$ & $-0.103(-0.110,-0.096)$ & $-0.094(-0.101,-0.087)$ & $-0.105(-0.112,-0.097)$ \\
\hline Total cholesterol/HDL-C & $0.323(0.294,0.352)$ & $0.352(0.323,0.381)$ & $0.327(0.298,0.356)$ & $0.371(0.342,0.400)$ \\
\hline Triglycerides, $\mathrm{mmol} / \mathrm{l}^{\mathrm{a}}$ & $0.119(0.111,0.127)$ & $0.141(0.134,0.149)$ & $0.139(0.131,0.147)$ & $0.148(0.140,0.156)$ \\
\hline Systolic BP, mm Hg & $4.02(3.78,4.26)$ & $3.54(3.30,3.79)$ & $2.04(1.79,2.29)$ & $3.50(3.25,3.74)$ \\
\hline Diastolic BP, mm Hg & $2.64(2.48,2.79)$ & $2.46(2.30,2.61)$ & $1.48(1.31,1.64)$ & $2.43(2.28,2.59)$ \\
\hline $\mathrm{CRP}, \mathrm{mg} / \mathrm{l}$ & $0.425(0.407,0.443)$ & $0.408(0.389,0.426)$ & $0.275(0.256,0.294)$ & $0.427(0.409,0.446)$ \\
\hline Fibrinogen, g/1 & $0.162(0.147,0.176)$ & $0.166(0.152,0.181)$ & $0.105(0.090,0.120)$ & $0.172(0.157,0.187)$ \\
\hline
\end{tabular}

$\mathrm{BP}=$ Blood pressure

${ }^{a}$ Natural logarithm.

index. Our study is thus relevant to the ongoing debate about the definition of MS [8, 10, 16-18]. The identification of MS may be particularly relevant in women, not only because it is strongly predictive of type 2 diabetes $[19,20]$ which occurs more commonly in women than men, but also because its presence, even before the onset of diabetes, removes much of the protection against CVD enjoyed by women compared to men [21, 22]. Our study justifies the use of some measure of central obesity in the definition of MS. However, WC, the most frequently proposed indicator of visceral obesity for the definition of MS [10-12, 16-18] was less closely associated with the metabolic and inflammatory risk markers than the ratio between WC and the women's height. This suggests that taking height into account might improve the definition of MS based on WC. Our study does add substantially to earlier evidence for this view [23-25]. The overall improvement in the definition of MS from the addition of height to WC might seem small. However, it will be of much greater importance in women at the extremes of the height distribution. Intuitively, it seems inappropriate to base an estimate of central obesity solely on WC which is going to be higher, independently of the presence of visceral fat, in taller as opposed to shorter women. This has already posed difficulties in reaching agreement about a global definition of MS, because Asian women tend to be shorter than those of European or African descent [6]. It is particularly important that any agreed definition should be equally valid in Asia where coronary heart disease is rapidly emerging as nutritional changes take place [3]. The use of WHTR might offer the prospect of a more satisfactory global definition. It must, however, be conceded that the additional measurement of height might make doctors and nurses less prepared to identify MS. However, their greater reluc- 
tance seems to be to measure WC rather than height which is widely used in the more traditional measurement of BMI. Currently, recommendations for the definition of MS in women are divided as to whether a WC of $\geq 88$ or $>80 \mathrm{~cm}$ should be the criterion for central obesity [8-12]. Based on our data, we would propose that a WHTR of 0.54 or 0.50 , respectively replaces the WC in the definition of MS These WHTRs are obtained by dividing 88 or $80 \mathrm{~cm}$, respectively by the average height of women in England which is $161.4 \mathrm{~cm}$ [15]. Both are close to the mean WHTR in women of all ages in our study population, but then so are the figures of 80 and $88 \mathrm{~cm}$ for WC used in the currently proposed definition of MS. This, of course, does not mean to imply that around half of British women have MS, because in addition they would be required to have at least 2 other cardiovascular risk factors.

Blood pressure is the one cardiovascular risk factor which does not relate most closely to either WHTR or WC; rather its closest association is with BMI. It has been noted previously that blood pressure is more strongly influenced by obesity generally [26] rather than by indices of insulin resistance such as the insulin concentration or measures of central obesity [27, 28]. It has been difficult to relate change in blood pressure to insulin, insulin resistance or non-esterified fatty acid levels [29, 30] which seem to mediate the dyslipidaemia and increased fibrinogen and CRP in MS. Recently, it was proposed that the association of obesity with blood pressure may be at least partly due to increased sympathetic activity. Mechanistically, this may result from increased leptin release which occurs from adipose tissue generally, and not specifically from visceral adipose tissue [30].
There were marked effects of age on serum cholesterol, LDL$\mathrm{C}$, triglycerides, CRP, plasma fibrinogen and blood pressure in our large cohort of healthy women employees of a large department store chain, living throughout the UK. Serum HDL$\mathrm{C}$ concentration in this non-diabetic group of women remained constant with respect to age. There is close agreement between the population distributions of values for serum cholesterol, LDL-C, HDL-C, triglycerides, blood pressure and plasma fibrinogen reported here and those reported in the smaller Health Survey for England (HSE) [15]. The lower CRP levels measured in our study compared to the HSE, particularly in the younger women, are likely to be the result of our exclusion of women receiving oestrogen-containing preparations which are known to raise CRP levels [31]. The relative lack of effect of age on mean levels of HDL-C has been reported previously $[14,15,32]$. Only a minority of studies have suggested any marked change at the time of the menopause in non-diabetic women [33].

We conclude that the definition of MS may be improved by adjustment of WC for height using a simple ratio, and that HDL cholesterol, unlike the other cardiovascular risk factors evaluated here, is unaffected by advancing age in non-diabetic women.

\section{Acknowledgments}

This study was supported by the Simon Warburg Voluntary Reserve Fund, the Lipid Diseases Fund and the NHS R and D Levy. SEH is supported by the British Heart Foundation (RG 2005/014).

\section{References}

1 Castelli WP: Epidemiology of coronary heart disease: the Framingham study. Am J Med 1984;76: 4-12.

2 Barrett-Connor E: Sex differences in coronary heart disease. Why are women so superior? The 1995 Ancel Keys Lecture. Circulation 1997;95: 252-264.

3 Mackay J, Mensah GA: The Atlas of Heart Disease and Stroke. Geneva, WHO, 2004, pp 30-31.

4 Office for National Statistics (1998): Prevalence of treated coronary heart disease per 1000 patients, by age, sex and deprivation category $1994-98$. www. statistics. gov.uk (accessed 21.03.07)

5 Larsson B, Bengtsson C, Bjorntorp P, Lapidus L, Sjostrom L, Svardsudd K, Tibblin G, Wedel H, Welin L, Wilhelmsen L: Is abdominal body fat distribution a major explanation for the sex difference in the incidence of myocardial infarction? The study of men born in 1913 and the study of women, Goteborg, Sweden. Am J Epidemiol 1992;135: 266-273.

6 Lemieux S, Despres JP, Moorjani S, Nadeau A, Theriault G, Prud'home D, Tremblay A, Bouchard C, Lupien PJ: Are gender differences in cardiovascular disease risk factors explained by the level of visceral adipose tissue? Diabetologia 1994;37:757-764.
7 Kannel WB, Wilson PWF, Nam BH, D'Agostino RB: Risk stratification of obesity as a coronary risk factor. Am J Cardiol 2002;90:697-701.

8 Alberti KG, Zimmet P, Shaw J; IDF Epidemiology Task Force Consensus Group: The metabolic syndrome: a new worldwide definition. Lancet 2005; 366:1059-1062.

$\checkmark 9$ Grundy SM: Metabolic syndrome: connecting and reconciling cardiovascular and diabetes worlds. J Am Coll Cardiol 2006;47:1093-1100.

10 Expert panel on Detection, Evaluation, and Treatment of High Blood Cholesterol in Adults: Executive summary of the third report of the National Cholesterol Education Program (NCEP) expert panel on detection, evaluation, and treatment of high blood cholesterol in adults (Adult Treatment Panel III). JAMA 2001;285:2486-2497.

11 Wood DA, Wray R, Poulter N, Williams B, Kirby M, Patel V, Durrington P, Reckless J, Davies M, Sivers F, Potter J: JBS2: Joint British guidelines on prevention of cardiovascular disease in clinical practice. Heart 2005;91(suppl V):v1-52.

12 De Backer G, Ambrosioni E, Borch-Johnsen K, Brotons C, Cifkova R, Dallongeville J, Ebrahim S, Faergeman O, Graham I, Mancia G, Cats VM, Orth-Gomer K, Perk J, Pyorala K, Rodicio JL, Sans S, Sansoy V, Sechtem U, Silber S, Thomsen T, Wood
D: European guidelines on cardiovascular disease prevention in clinical practice. Third Joint Task Force of European and other Societies on Cardiovascular Disease Prevention in Clinical Practice (constituted by representatives of eight societies and by invited experts). Atherosclerosis 2004;173: 381-391.

13 Friedewald WT, Levy RI, Fredrickson DS: Estimation of the concentration of low-density lipoprotein cholesterol in plasma, without use of the preparative ultracentrifuge. Clin Chem 1972;18:499-502.

14 Rifkind BM, Segal P: Lipid research clinics program reference values for hyperlipidemia and hypolipidemia. JAMA 1983;250:1869-1972.

15 Spriston K, Primatesta P (eds): Risk factors for cardiovascular disease. Health Survey for England 2003, vol. 2. London, The Stationery Office, 2004.

16 Greenland P: Critical questions about the metabolic syndrome. Circulation 2005;112:3675-3676.

17 Grover SA, Coupal L, Hu X-P: Identifying adults at increased risk of coronary disease. How well do the current cholesterol guidelines work? JAMA 1995;274:801-806.

18 Sattar N: The metabolic syndrome: should current criteria influence clinical practice? Curr Opin Lipidol 2006;17:404-411. 
19 Hanley AJ, Karter AJ, Williams K, Festa A, D'Agostino RBJr, Wagenknecht LE, Haffner SM: Prediction of type 2 diabetes mellitus with alternative definitions of the metabolic syndrome: the Insulin Resistance Atherosclerosis Study. Circulation 2005;112:3713-3721.

20 Wilson PFW, D'Agostino RB, Parise H, Sullivan L, Meigs JB: Metabolic syndrome as a precursor of cardiovascular disease and type 2 diabetes mellitus. Circulation 2005;112:3066-3072.

21 Haffner SM, Stern MP, Hazuda HP, Mitchell BD, Patterson JK: Cardiovascular risk factors in confirmed prediabetic individuals: does the clock for coronary disease start ticking before the onset of clinical diabetes. JAMA 1990;263:2893-2898.

22 Barrett-Connor EL, Cohn BA, Wingaard DL, Edelstein SL: Why is diabetes mellitus a stronger risk factor for fatal ischaemic heart disease in women than in men? The Rancho Benardo Study. J Am Med Assoc 1991;265:627-631.

23 Hsieh SD, Yoshinaga H, Muto T: Waist-to-height ratio, a simple and practical index for assessing central fat distribution and metabolic risk in Japanese men and women. Int J Obes Relat Metab Disord 2003;27:61-66.
24 Mannucci E, Alegiani SS, Monami M, Sarli E, Avogaro A; DAI (Diabetes and Informatics) Study Group: Indexes of abdominal adiposity in patients with type 2 diabetes. J Endocrinol Invest 2004;27: 535-540.

25 Schneider HJ, Glaesmer H, Klotsche J, Bohler S, Lehnert H, Zeiher AM, Marz W, Pittrow D, Stalla GK, Wittchen HU: Accuracy of anthropometric indicators of obesity to predict cardiovascular risk. J Clin Endocrinol Metab 2007;92:589-594.

26 Stamler R, Stamler J, Riedlinger WF, Algera G, Roberts RH: Weight and blood pressure. Findings in hypertension screening of 1 million Americans. JAMA 1978;240:1607-1610.

27 Wannamethee SG, Shaper AG, Durrington PN, Perry IJ: Hypertension, serum insulin, obesity and the metabolic syndrome. J Human Hypertension 1998;12:735-741.

28 Wyszynski DF, Waterworth DM, Barter PJ, Cohen J, Kesaniemi YA, Mahley RW, McPherson R, Waeber G, Bersot TP, Sharma SS, Nolan V, Middleton LT, Sundseth SS, Farrer LA, Mooser V, Grundy SM: Relation between atherogenic dyslipidemia and the Adult Treatment Program-III definition of metabolic syndrome (Genetic Epidemiology of Metabolic Syndrome Project). Am J Cardiol 2005;95: 194-198.
29 Rocchini AP, Yang JQ, Gokee A: Hypertension and insulin resistance are not directly related in obese dogs. Hypertension 2004;43:1011-1016.

30 Rahmouni K, Correia MLG, Haynes WG, Mark AL: Obesity-associated hypertension. Hypertension 2005;45:9-14.

31 Pradhan AD, Manson JE, Rossouw JE, Siscovick DS, Mouton CP, Rifai N, Wallace RB, Jackson RD Pettinger MB, Ridker PM: Inflammatory biomarkers, hormone replacement therapy, and incident coronary heart disease: prospective analysis from the Women's Health Initiative observational study. JAMA 2002;288:980-987.

32 Razay G, Heaton KW, Bolton CH: Coronary heart disease risk factors in relation to menopause Q J Med 1992;85:307-308

33 Stevenson JC, Crook D, Godsland IF: Effects of age and menopause on lipid metabolism in healthy women. Atherosclerosis 1993;98:83-90. 